\title{
A Case of Atrial Flutter Masking Acute Pericarditis
}

\author{
Christopher Schwartz ${ }^{1}$, Arjun C. Khadilkar $^{1}$, Christopher Bitetzakis ${ }^{2}$, Aarti Patel ${ }^{2}$ \\ 1. Internal Medicine, University of South Florida Morsani College of Medicine, Tampa, USA 2. Cardiology, University of \\ South Florida Morsani College of Medicine, Tampa, USA
}

Corresponding author: Arjun C. Khadilkar, akhadilkar18@gmail.com

\begin{abstract}
Atrial flutter (AFL) is a macro-reentrant tachycardia that can be provoked by numerous factors, including acute pericarditis. We present a case of new-onset AFL masking acute pericarditis in a man with multiple comorbid conditions, including hypertension, chronic kidney disease, and obstructive sleep apnea. After a failed attempt of rate control, the patient underwent successful cardioversion, which revealed electrocardiographic findings consistent with acute pericarditis. Colchicine was avoided in the setting of chronic kidney disease and the patient was treated with a steroid taper. Pericarditis is a rare cause of AFL, and this case demonstrates the diagnostic and management considerations for AFL and acute pericarditis.
\end{abstract}

Categories: Cardiology, Internal Medicine, Medical Education

Keywords: atrial flutter, acute pericarditis, cardioversion, ekg, supraventricular tachycardia

\section{Introduction}

Atrial flutter (AFL) is an arrhythmia characterized by rapid, regular, atrial depolarizations at a characteristic rate of between 250 and 350 beats per minute with a $2: 1$ relation between atrial and ventricular activity [1]. Typical flutter is defined by a macro-reentrant circuit around the cavotricuspid isthmus (CTI), generally in a counterclockwise rotation. Atypical flutter occurs outside the CTI around areas of atrial scar tissue. On electrocardiogram (ECG), typical p-waves are absent, and the atrial activity is seen in a sawtooth pattern known as "F waves" in leads II, III, and aVF [2]. Clinical presentation is often dependent on the ventricular rate and can lead to dyspnea, fatigue, palpations, and lightheadedness. AFL is uncommon in structurally normal hearts and can be seen in clinical contexts similar to those presenting with atrial fibrillation.

Pericarditis is a known trigger for cardiac arrhythmias [3,4]. It is characterized by pericardial sac inflammation due to numerous causes such as infectious, neoplastic, inflammatory, autoimmune, metabolic, pharmacologic, or toxic. On ECG, there may be widespread concave ST elevations and PR depressions with reciprocity in aVR. Presentation varies depending on the underlying etiology but often includes chest pain characterized as sharp, pleuritic, and improved by leaning forward. A pericardial friction rub may be heard during auscultation [5].

Review began 03/23/2021 Review ended 03/25/2021 Published 03/29/2021

\section{๑) Copyright 2021}

Schwartz et al. This is an open access article distributed under the terms of the Creative Commons Attribution License CC-BY 4.0., which permits unrestricted use, distribution, and reproduction in any medium, provided the original author and source are credited.
We present a case of an 83-year-old-man with supraventricular tachycardia determined to be AFL status post an adenosine trial. After a failed attempt at rate control, the patient underwent cardioversion. ECG findings post-cardioversion in normal sinus rhythm were consistent with pericarditis, which was likely the cause for his new-onset AFL.

\section{Case Presentation}

An 83 year-old-male with hypertension, stage three chronic kidney disease, hypothyroidism, transient ischemic attack, and sleep apnea presented with three weeks of pleuritic chest pain, fatigue, subjective fevers, and progressive shortness of breath. The initial ECG was significant for supraventricular tachycardia interpreted as new-onset atrial fibrillation with a rapid ventricular response.

Labs were negative for electrolyte abnormalities and creatinine was $1.5 \mathrm{mg} / \mathrm{dL}$ (baseline $1.5-1.9 \mathrm{mg} / \mathrm{dL}$ ). Troponin-I was within normal limits at $0.01 \mathrm{ng} / \mathrm{mL}$. Influenza A, B, and respiratory syncytial virus (RSV) nasal polymerase chain reaction (PCR) were negative. Coronavirus 2019 (COVID-19) nasal rapid PCR was negative. Thyroid-stimulating hormone was within normal limits at $1.902 \mathrm{lU} / \mathrm{ml}$. C-reactive protein, fibrinogen, and brain natriuretic peptide (BNP) were all elevated at $8.3 \mathrm{mg} / \mathrm{dL}, 583 \mathrm{mg} / \mathrm{dl}, 182 \mathrm{pg} / \mathrm{mL}$, respectively. A trans-thoracic echocardiogram showed a preserved ejection fraction of $55-60 \%$ with moderate concentric hypertrophy without pericardial effusion. The patient initially received boluses of intravenous diltiazem and transitioned briefly to a continuous diltiazem infusion, which was discontinued due to hypotension. He received an amiodarone load intravenously for rhythm control and renally dosed apixaban for a $\mathrm{CHA}_{2} \mathrm{DS}_{2}$-VASc score of five. Short-acting intravenous diltiazem was restarted and uptitrated for adequate rate control with hemodynamic stability. 


\section{Cureus}

Adenosine was subsequently administered and revealed AFL with variable AV block. A typical atrial activity has been highlighted in Figure 1, and the ECG in Figure 2 highlights the atrial activity in the patient.

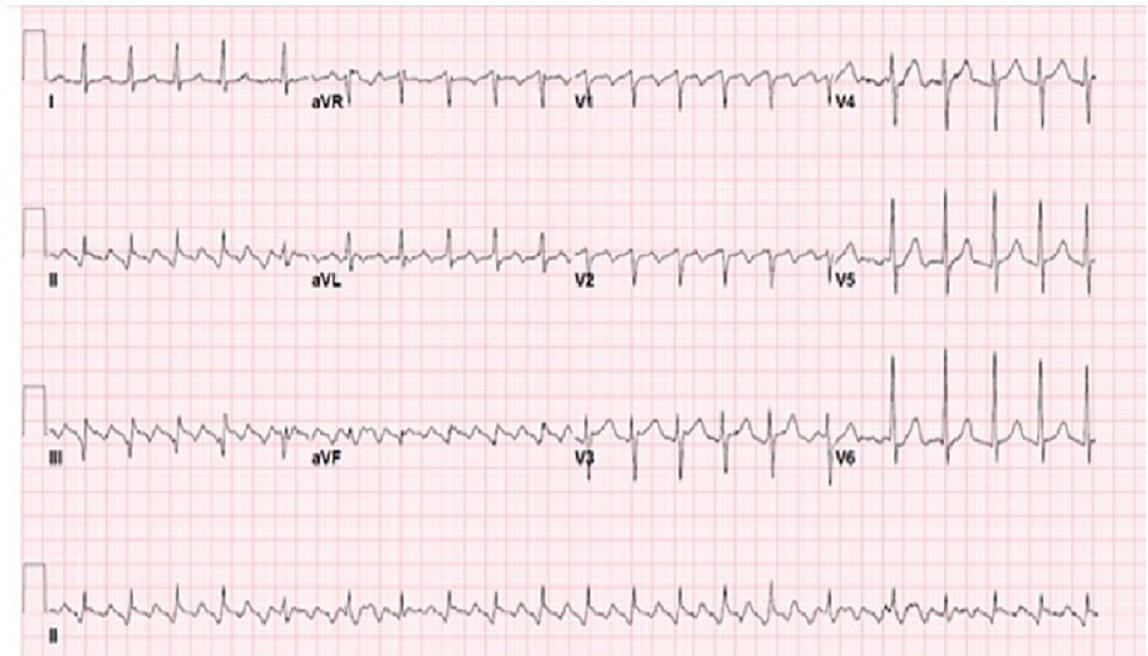

FIGURE 1: Typical atrial flutter with variable AV block

AV block: atrioventricular block [6].

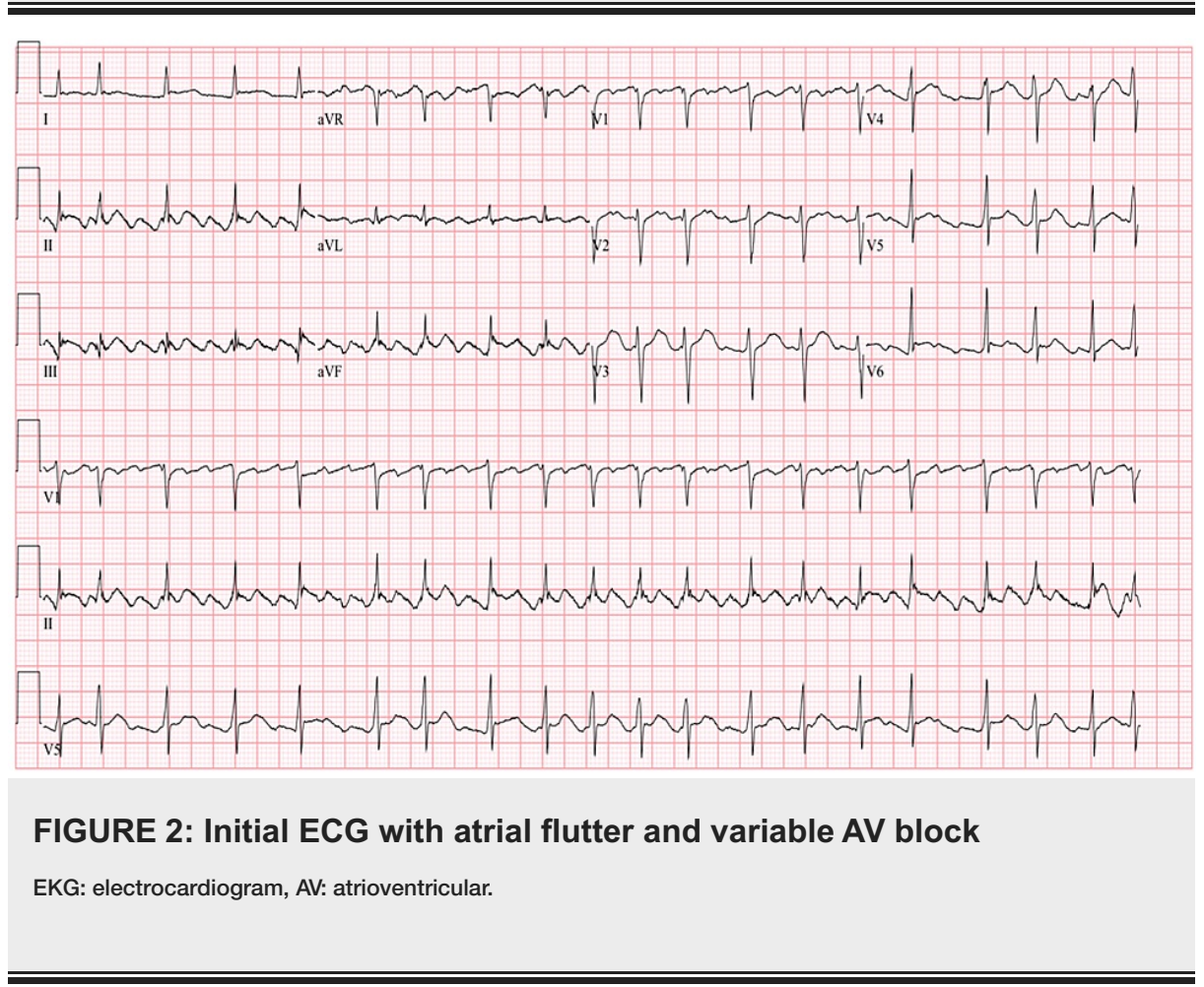

Since the patient had persistently increased rates despite initial rate and rhythm control strategies, cardioversion was considered for rhythm control. Intracardiac thrombi were excluded via transesophageal echocardiography and the patient underwent successful electrical cardioversion. Typically, acute pericarditis presents with widespread concave ST elevations and PR depressions with reciprocity in aVR, highlighted in Figure 3. A post-cardioversion 12 lead ECG demonstrated normal sinus rhythm with ST elevations present in the precordial leads with concomitant T-wave abnormalities in V2 through V6, limb leads I, II, and aVL. PRsegment depression was seen in leads I, II, V2, V3, and ST depression in lead aVR (Figure 4). 


\section{Cureus}

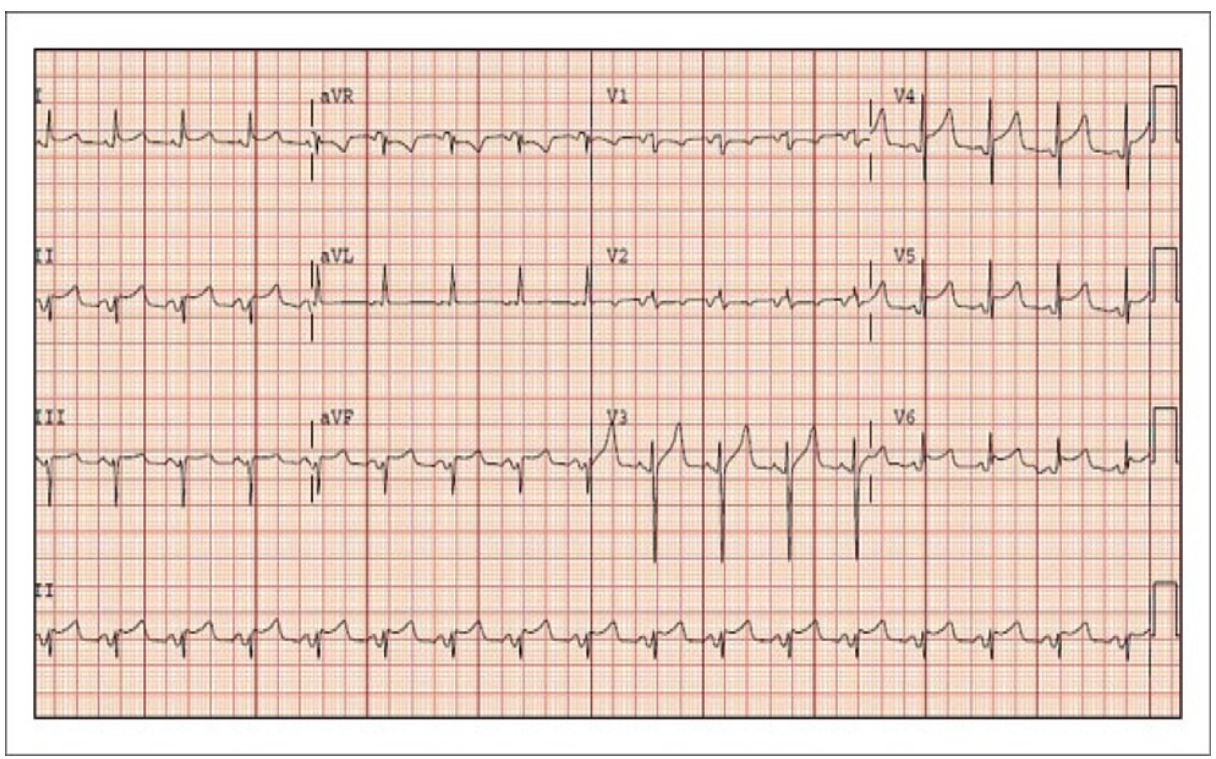

\section{FIGURE 3: Widespread, concave, ST elevations and PR depressions with reciprocity in aVR}

aVR: augmented vector right [7].

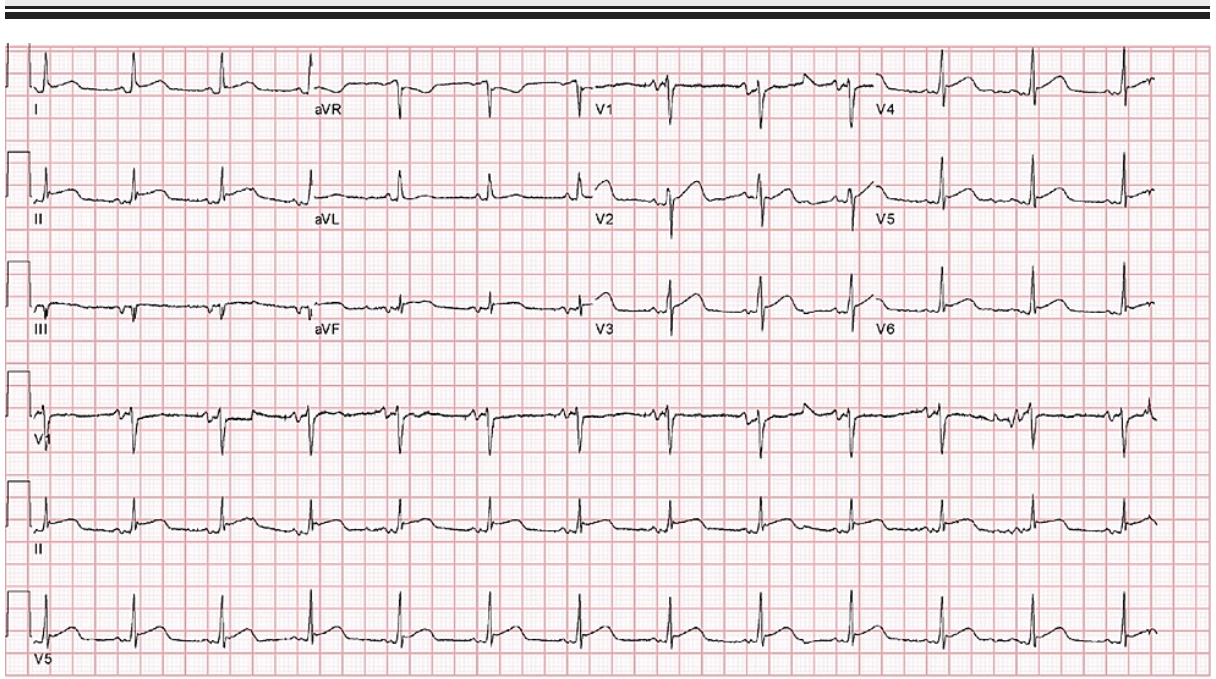

FIGURE 4: Post-cardioversion ECG consistent with acute pericarditis

ECG: electrocardiogram.

The patient was asymptomatic post-cardioversion without chest pain, shortness of breath, or diaphoresis He was diagnosed with pericarditis and treated with a steroid taper due to his chronic kidney disease and history of gastrointestinal bleed in the setting of non-steroidal anti-inflammatory agent (NSAID) use.

\section{Discussion}

In one population-based investigation of AFL, among 58,820 patients in central Wisconsin, those at highest risk for developing AFL were patients with heart failure and chronic obstructive pulmonary disease, with the arrhythmia being uncommon in structurally normal hearts [8]. AFL occurs in similar clinical causes as atrial fibrillation, including but not limited to pericarditis. Though data related to the incidence of arrhythmias in the setting of pericarditis are sparse, atrial fibrillation appears to be the most common across studies occurring at rates ranging from $4.3 \%$ to $25 \%$ [9]. This wide range is likely due to the various pericarditis etiologies studied and researcher variation in the definition of what constituted a supraventricular arrhythmia. It is unclear why pericarditis predisposes those to arrhythmias though some suggest that the proximity of an inflammatory process to the sinus node may be arrhythmogenic; however, postmortem investigations have challenged this presumption [10]. 
In the western world, most pericarditis cases are diagnosed as idiopathic after negative work-up, though many of these cases are presumed to be viral [11]. Among immunocompetent patients in developed countries, acute pericarditis is assumed to be of viral etiology with NSAIDs as the mainstay of therapy. Interestingly, the efficacy of NSAIDs has only been evaluated in one randomized control trial with patients suffering from post-pericardiotomy syndrome [12]. Additionally, evidence supports the use of colchicine as an adjunct therapy to NSAIDs to improve remissions rates in both acute and recurrent pericarditis [13].

In those with chronic kidney disease that have contraindications to NSAIDs or colchicine, steroid therapy can be used for the treatment of pericarditis but is associated with disease recurrence. This is especially true early in the disease course as seen in the Colchicine in Addition to Conventional Therapy for Acute Pericarditis (COPE) trial and in a subsequent systematic review where glucocorticoids were a significant predictor of recurrence [13,14]. There is also evidence from animal studies that glucocorticoids may exacerbate virally induced pericarditis by effects on virus replication [15]. Thus, glucocorticoids may perpetuate pericardial inflammation and are considered second-line therapy per the European Society of Cardiology (ESC) [16]. When used, the ESC recommends low to moderate doses such as prednisone 0.2-0.5 $\mathrm{mg} / \mathrm{kg} /$ day until resolution of symptoms or normalization of C-reactive protein, at which point tapering should begin.

\section{Conclusions}

Atrial fibrillation and AFL are provoked by similar and diverse pathologies including pericarditis. AFL can be managed through pharmacological, electrical, or ablative means. In our case, initial pharmacotherapy treatment failed. After successful electrical cardioversion, a 12-lead ECG demonstrated acute pericarditis, which was the most likely trigger for AFL. Considering his comorbidities of chronic kidney disease and history of NSAID-induced GI bleeding, we concluded that a steroid taper with serial inflammatory laboratory monitoring was the most appropriate therapeutic option moving forward. Pericarditis rarely triggers AFL and, as seen in this case, the classic ECG features of acute pericarditis can be masked during episodes of typical AFL. This case demonstrates the inpatient management considerations for AFL and alternative therapeutic modalities for those with pericarditis and contraindications to first-line therapy.

\section{Additional Information \\ Disclosures}

Human subjects: Consent was obtained or waived by all participants in this study. Conflicts of interest: In compliance with the ICMJE uniform disclosure form, all authors declare the following: Payment/services info: All authors have declared that no financial support was received from any organization for the submitted work. Financial relationships: All authors have declared that they have no financial relationships at present or within the previous three years with any organizations that might have an interest in the submitted work. Other relationships: All authors have declared that there are no other relationships or activities that could appear to have influenced the submitted work.

\section{References}

1. Wellens HJ: Contemporary management of atrial flutter. Circulation. 2002, 106:649-52. 10.1161/01.cir.0000027683.00417.9a

2. Cosío FG: Atrial flutter, typical and atypical: a review . Arrhythm Electrophysiol Rev. 2017, 6:55-62. 10.15420/aer.2017.5.2

3. Spodick DH: Frequency of arrhythmias in acute pericarditis determined by Holter monitoring . Am J Cardiol. 1984, 53:842-5. 10.1016/0002-9149(84)90416-8

4. Spodick DH: Arrhythmias during acute pericarditis. A prospective study of 100 consecutive cases . JAMA. 1976, 235:39-41. 10.1001/jama.1976.03260270025020

5. Abuarqoub A, Elshimy G, Shittu M, Hamdan A, Shamoon F: Right atrial fibroelastoma presenting as typical atrial flutter: rare disease in unusual location. Case Rep Cardiol. 2017, 2017:4764587. 10.1155/2017/4764587

6. Masek KP, Levis JT: ECG diagnosis: acute pericarditis . Perm J. 2013, 17 :e146. 10.7812/TPP/13-044

7. Imazio M, Gaita F, LeWinter M: Evaluation and treatment of pericarditis: a systematic review . JAMA. 2015, 314:1498-506. 10.1001/jama.2015.12763

8. Granada J, Uribe W, Chyou PH, et al.: Incidence and predictors of atrial flutter in the general population . J Am Coll Cardiol. 2000, 36:2242-6. 10.1016/s0735-1097(00)00982-7

9. Imazio M, Lazaros G, Picardi E, et al.: Incidence and prognostic significance of new onset atrial fibrillation/flutter in acute pericarditis. Heart. 2015, 101:1463-7. 10.1136/heartjnl-2014-307398

10. Colas C: The normal and pathological sinusal node. Nouv Presse Med. 1979, 8:4118.

11. Kytö V, Sipilä J, Rautava P: Clinical profile and influences on outcomes in patients hospitalized for acute pericarditis. Circulation. 2014, 130:1601-6. 10.1161/CIRCULATIONAHA.114.010376

12. Horneffer PJ, Miller RH, Pearson TA, Rykiel MF, Reitz BA, Gardner TJ: The effective treatment of postpericardiotomy syndrome after cardiac operations. A randomized placebo-controlled trial. J Thorac Cardiovasc Surg. 1990, 100:292-6.

13. Imazio M, Bobbio M, Cecchi E, et al.: Colchicine in addition to conventional therapy for acute pericarditis: results of the COlchicine for acute PEricarditis (COPE) trial. Circulation. 2005, 112:2012-6. 10.1161/CIRCULATIONAHA.105.542738

14. Lotrionte M, Biondi-Zoccai G, Imazio M, et al.: International collaborative systematic review of controlled clinical trials on pharmacologic treatments for acute pericarditis and its recurrences. Am Heart J. 2010, 


\section{Cureus}

160:662-70. 10.1016/j.ahj.2010.06.015

15. Kilbourne E, Wilson C, Perrier D: The induction of gross myocardial lesions by a Coxsackie (pleurodynia) virus and cortisone. J Clin Invest. 1956, 35:362-70. 10.1172/JCI103286

16. Adler Y, Charron P, Imazio M, et al.: [2015 ESC Guidelines for the diagnosis and management of pericardial diseases]. Kardiol Pol. 2015, 73:1028-91. 10.5603/KP.2015.0228 